\title{
SMART GRIDS COMO ALTERNATIVA DE DESENVOLVIMENTO URBANO INTELIGENTE E SUSTENTÁVEL EM PARINTINS/AM
}

\author{
Jerrylee Dilson Yoshii Silva ${ }^{1}$ \\ Universidade Federal do Amazonas, Amazonas, Brasil. \\ jerrylee ni@hotmail.com \\ Thalita Reis da Silva ${ }^{2}$ \\ https://orcid.org/0000-0002-0216-2738 \\ Universidade Federal do Amazonas, Amazonas, Brasil. \\ thalitarsilva@ufam.edu.br \\ Erick de Freitas Moura ${ }^{3}$ \\ https://orcid.org/0000-0003-1432-8827 \\ Universidade Federal do Amazonas, Amazonas, Brasil.
}

erickdfreitas@ufam.edu.br

Para citar este artículo puede utilizar el siguiente formato:

Jerrylee Dilson Yoshii Silva, Thalita Reis da Silva y Erick de Freitas Moura: "Smart Grids como alternativa de desenvolvimento urbano inteligente e sustentável em Parintins/AM", Revista Caribeña de Ciencias Sociales (vol 10, № 8 octubre-diciembre 2021, pp. 176-191. En línea:

https://doi.org/10.51896/caribe/GSEO6731

\section{RESUMO}

Devido à rápida e não planejada urbanização surgem problemas de aclimatação urbana, para os quais alternativas de soluções foram desenvolvidas a partir do conceito de Smart City. Nesta pesquisa, o foco é nos problemas da gestão da produção e da distribuição de energia elétrica em Parintins/AM. Para a problemática energética são utilizados os conceitos de rede elétrica inteligente, Smart Grid, que por meio da tecnologia otimiza os sistemas de produção e distribuição de energia, tornando-os mais eficientes, eficazes e sustentáveis. O objetivo deste trabalho é analisar, com base nas teorias desenvolvidas acerca das Smart Cities e das Smart Grids, quais são os aspectos que devem ser levados em consideração para a gestão inteligente e sustentável da produção e distribuição de energia elétrica em Parintins/AM. O paradigma de pesquisa é o funcionalista, por meio de pesquisa qualitativa, com delineamento descritivo e analítico. Os dados foram levantados

\footnotetext{
${ }^{1}$ Bacharelando em Administração pela Universidade Federal do Amazonas (UFAM).

${ }^{2}$ Doutora em Propriedade Industrial e Inovação, pelo Instituto Nacional de Propriedade Industrial (INPI). Administradora de empresas pela Universidade Federal do Amazonas (UFAM) e especialista em Administração Pública, também pela UFAM. Mestre pela Universidade Federal de Rondônia (UNIR), em gestão, com ênfase em liderança. Professora Adjunta da Universidade Federal do Amazonas, no curso de Administração, no município de Parintins/AM.

${ }^{3}$ Professor Assistente na Universidade Federal do Amazonas (ICSEZ/UFAM). Graduado e Mestre em Administração pela Universidade Federal de Uberlândia (FAGEN/UFU). Doutorando pela Universidade Estadual de Campinas, na Faculdade de Ciências Aplicadas (FCA/UNICAMP). Membro do SB-Lab - Laboratório de Negócios Sustentáveis (FCA/UNICAMP). Áreas de interesse em pesquisa: Cidades Inteligentes, Sustentabilidade, Telemedicina e Estratégia.
} 
por meio de revisão bibliográfica, análise documental e entrevistas semiestruturadas, triangulados e analisados mediante técnica de análise de conteúdo. Os resultados obtidos foram: 1) desenvolvimento científico na área das Smart Cities e Smart Grids, em construção, por meio de estudo empírico no interior do Amazonas; 2) identificação dos fatores impulsionadores e limitantes à implantação de Smart Grids em Parintins/AM.

Palavras-chave: Smart Cities; Smart Grids; Gestão de serviços; Sustentabilidade.

\title{
LAS REDES INTELIGENTES COMO ALTERNATIVA PARA EL DESARROLLO URBANO INTELIGENTE Y SOSTENIBLE EN PARINTINS/AM
}

\section{RESUMEN}

Debido a la urbanización rápida y no planificada, surgen problemas de aclimatación urbana, para lo cual se desarrollaron soluciones alternativas basadas en el concepto de Smart City. En esta investigación, la atención se centra en los problemas de gestión de la producción y distribución de electricidad en Parintins/AM. Para temas energéticos se utilizan los conceptos de red inteligente, Smart Grid, que a través de la tecnología optimiza los sistemas de producción y distribución de energía, haciéndolos más eficientes, efectivos y sostenibles. El objetivo de este trabajo es analizar, a partir de las teorías desarrolladas sobre Smart Cities y Smart Grids, cuáles son los aspectos que deben tenerse en cuenta para la gestión inteligente y sostenible de la producción y distribución de energía eléctrica en Parintins/AM. El paradigma de la investigación es el funcionalista, a través de la investigación cualitativa, con diseño descriptivo y analítico. Los datos fueron recolectados mediante revisión de literatura, análisis de documentos y entrevistas semiestructuradas, triangulados y analizados mediante la técnica de análisis de contenido. Los resultados obtenidos fueron: 1) desarrollo científico en el área de Smart Cities y Smart Grids, en construcción, a través de un estudio empírico en el interior de Amazonas; 2) identificación de factores impulsores y limitantes para la implementación de Smart Grids en Parintins/AM.

Palabras Ilave: Smart Cities; Smart Grids; Gestión de Servicios; Sustentabilidad.

\section{SMART GRIDS AS AN ALTERNATIVE FOR SMART AND SUSTAINABLE URBAN DEVELOPMENT IN PARINTINS/AM}

\begin{abstract}
Due to the rapid and unplanned urbanization, urban acclimatization problems arise, for which alternative solutions were developed based on the Smart City concept. In this research, the focus is on the problems of managing the production and distribution of electricity in Parintins/AM. For energy issues, the concepts of Smart Grid are used, which through technology optimizes energy production and distribution systems, making them more efficient, effective, and sustainable. The objective of this work is to analyze, based on the developed theories about Smart Cities and Smart Grids, which are the aspects that must be considered for the intelligent and sustainable management of the production and distribution of electric energy in Parintins/AM. The research paradigm is the functionalist one,
\end{abstract}


through qualitative research, with descriptive and analytical design. Data were collected through literature review, document analysis and semi-structured interviews, triangulated and analyzed using the content analysis technique. The results obtained were: 1) scientific development in the area of Smart Cities and Smart Grids, under construction, through an empirical study in the interior of Amazonas; 2) identification of driving and limiting factors for the implementation of Smart Grids in Parintins/AM.

Keywords: Smart Cities; Smart Grid; Service management; Sustainability.

\section{INTRODUÇÃO}

O município de Parintins, localizado a $369 \mathrm{~km}$ da capital Manaus/AM, possui aproximadamente 115.363 (cento e quinze mil, trezentos e sessenta e três) habitantes (IBGE, 2020) e é conhecido como "llha Encantada", devido ao maior espetáculo de ópera a céu aberto da América Latina, o festival folclórico dos Bois-Bumbá Caprichoso, lado azul, e Garantido, lado vermelho (Lima et al., 2013). Não obstante ser a segunda maior cidade em termos populacionais do Estado do Amazonas, Parintins possui infraestrutura urbana precária e o título de "Ilha Encantada" abrange tão somente a criatividade da população local para a elaboração do Festival Folclórico do Boi-Bumbá, aspecto considerado por Gil-Garcia, Pardo \& Nam (2015) como requisito para se desenvolver uma Smart City.

O investimento em infraestruturas e tecnologias inteligentes possibilita gerar grandes mudanças na economia, pois contribui com a modernização do atual e tradicional modelo econômico (Costa, 2014). Assim, os conceitos acerca das Smart Grids (Redes Inteligentes), abrangidos nos estudos das Smart Cities (Cidades inteligentes), são alternativas para auxiliar na gestão inteligente dos serviços de energia, por meio das Tecnologias da Informação e Comunicação (TICs), ao proporcionar otimização na produção, distribuição e consumo de energia elétrica de forma segura, eficiente e confiável (Rivera, Esposito \& Teixeira, 2013).

Parintins conta com o serviço de distribuição de energia feito pela Amazonas Distribuidora de Energia S.A, por meio da concessionária "Oliveira Energia" (ANEEL, 2019). Hodiernamente, a energia utilizada na cidade é a termoelétrica que, apesar de ter a vantagem de poder ser instalada em áreas habitadas, contribui para o aquecimento global ao ter como matéria-prima combustível fóssil (FRAGMAQ, 2015).

Devido à precariedade e não à sustentabilidade da gestão do sistema energético de Parintins/AM, esse estudo tem como objetivo analisar quais são os aspectos que devem ser levados em consideração para a gestão inteligente e sustentável da produção e distribuição de energia elétrica em Parintins/AM. A pergunta de pesquisa norteadora do estudo é "Como as Smart Grids se configuram em alternativa de desenvolvimento inteligente e sustentável em Parintins/AM?".

Com a finalidade de atingir o objetivo proposto, fez-se necessário fracioná-lo em objetivos específicos, os quais orientam os esforços para: 1) levantar os modelos de gestão inteligente de energia elétrica na literatura; 2) elaborar um modelo teórico de Smart Grid a partir da revisão da literatura; 3) descrever o modelo da gestão de energia elétrica em Parintins/AM; 4) elencar os fatores 
impulsionadores e limitantes à implantação de uma Smart Grid em Parintins/AM.

A temática das Smart Cities apresentou, nos últimos anos, aumento significativo na produção de trabalhos científicos (Cavalcante \& Tavares, 2017) e, por ser um campo de estudo ainda em construção, esta pesquisa contribui com o desenvolvimento deste campo científico, por meio de estudo aplicado na região amazônica. Ferreira et al. (2015) dizem que ao analisar os problemas e desafios de uma urbe de maneira integrada, é possível arranjar novos modos de construção de uma Smart City, a partir dos modelos já existente na literatura.

A justificativa prática da pesquisa advém da possibilidade da produção e da distribuição de energia elétrica de forma inteligente e sustentável, com reduções das interrupções em seu fornecimento e diminuição dos custos da energia comercializada, em Parintins/AM. Presumivelmente, o custo menor se configura, também, como justificativa social, que reflete em menores dispêndios financeiros nos consumos público e privado de energia, bem como no empresarial. Este último fator pode resultar na atenuação dos preços dos produtos e serviços praticados na ilha. Outrossim, Glaeser \& Berry (2006) consideram que os empreendedores estão se reunindo em centro urbanos inteligentes, pela disponibilidade de pessoas qualificadas e de infraestrutura urbana adequada para 0 desenvolvimento empresarial.

Isto posto, entende-se que o estudo acerca de cidades inteligentes, ao abordar um de seus aspectos, a energia inteligente, contribuirá para o desenvolvimento sustentável da região, bem como para reverter a situação de subemprego em Parintins, por elencar uma alternativa voltada à atração de empreendimentos para a cidade, além dos benefícios ambientais, ao se evitar a queima o de combustíveis fósseis na produção de energia elétrica.

\section{REVISÃO DE LITERATURA}

\subsection{Smart Cities: conceitos e abordagens}

Para a solução dos problemas advindos da rápida e não planejada expansão populacional urbana emergiram os conceitos das Smart Cities. As Smart Cities, Cidades Inteligentes, se refere a um conceito inovador, que tem sido aprimorado visando o melhor entendimento do desenvolvimento sustentável das cidades (Barbosa et al., 2013; Guedes et al., 2015).

Os gestores de cidades observaram que tem aumentado o grau de exigência da população por eficiência nos serviços públicos e na melhoria da qualidade de vida. Desta forma, verificou-se a necessidade de arranjar novas alternativas de administração, de forma que se atenda às necessidades da população e assegure a viabilidade futura e a prosperidade das cidades na perspectiva do tripé da sustentabilidade (Hollands, 2008; Chourabi, Nam \& Walker, 2012).

Para uma cidade se tornar smart e, consequentemente, proporcionar mais conforto para sua população, é necessário desenvolver as áreas de tecnologia, dados e informações, sustentabilidade ecológica, planejamento urbano, governança e prestação de serviços públicos. O desenvolvimento urbano em uma Smart City abrange, além das áreas de educação, saúde, moradia, saneamento básico e mão de obra qualificada, as áreas de gestão de resíduos, o uso de energia renovável e 
eficiente, uso inteligente da água, construções inteligentes, governança municipal e mobilidade urbana (Angelidou, 2014; Gil-Garcia, Pardo \& Nam, 2015; Ahvenniemi et al., 2017).

\subsection{Desenvolvimento Sustentável: Agenda 2030}

A Organização das Nações Unidas (ONU) tem como propósito "conseguir uma cooperação internacional para resolver os problemas internacionais de caráter econômico, social, cultural ou humanitário [...]", portanto, desde a fundação da ONU, em 1945, tem sido criadas diversas propostas para a promoção do desenvolvimento sustentável (ONU, 1945, p.5).

Dentre as ações da ONU teve-se a publicação do relatório de Brundtland, conhecido como "Nosso Futuro Comum" (Our Common Future), elaborado pela Comissão Mundial sobre o Meio Ambiente e Desenvolvimento, em 1987. Neste relatório foi dada uma definição para o termo "desenvolvimento sustentável" como "desenvolvimento que procura satisfazer as necessidades da geração atual, sem comprometer a capacidade das gerações futuras de satisfazerem as suas próprias necessidades", focando nas áreas de "população, segurança alimentar, perda de espécies e recursos genéticos, energia, indústria e assentamentos humanos" (WCED, 1987, p. 16-18).

Em setembro de 2015, a ONU criou a Agenda 2030 para o Desenvolvimento Sustentável (The 2030 Agenda for Sustainable Development). Esta agenda é um plano de ação formada por 17 Objetivos de Desenvolvimento Sustentável (ODS) e 169 metas e, dentre esses ODS, o décimo primeiro trata sobre as "Cidades e comunidades sustentáveis", com o objetivo de "tornar as cidades e os assentamentos humanos inclusivos seguros, resilientes e sustentáveis", e o sétimo aborda especificamente a temática de "Energia limpa e acessível", com objetivo "Assegurar o acesso confiável, sustentável, moderno e a preço acessível à energia para todos" (IPEA, 2019a; IPEA, 2019b).

O Brasil já é considerado um dos países líderes quanto ao acesso à energia elétrica, com expectativa de se tornar, até 2030 , o sexto país do mundo em termo de desenvolvimento de Smart Grids, com uma previsão de investimento inicial de 63 milhões em trocas de medidores inteligentes (Northeast-Group, 2012; Innovation-Observatory, 2012).

\subsection{Smart Grids: conceitos e abordagens}

De acordo com a International Energy Agency (IEA, 2011), a Smart Grid é um investimento que otimiza a infraestrutura física energética pelo uso eficiente das informações, criando sistemas capazes de se adaptarem quando do crescimento da demanda por energia elétrica. Segundo a Electric Power Research Institute a Smart Grid pode ser compreendida como: "um sistema que otimiza a fonte de alimentação e a entrega, minimiza as perdas, é auto reparável e permite a próxima geração de eficiência energética e aplicativos de resposta à demanda" (Alto, 2008, p. 19).

Os conceitos de Smart Grids abordam uma série de características inovadoras, portanto, a Smart Grid não deve ser compreendida somente como uma tecnologia ou equipamento específico. A Smart Grid visa a otimização do setor de energia elétrica e a integração das atividades dos usuários 
conectados por interfaces de comunicação, possibilitando economia de energia, redução de custos operacionais e aumento da confiança e transparência da prestação do serviço de energia. Devido à sua complexidade de implementação, faz-se necessário desenvolver "novas tecnologias, padrões, políticas e atividades regulatórias" (Falcão, 2009; Fan et al., 2013, p.1).

A Smart Grid é caracterizada pela implantação de diversos tipos de tecnologias dentro do sistema energia, como as TICs, sensoriamento, segurança cibernética, além de técnicas que possibilitem a operação integrada, otimizando o desempenho da rede e refletindo no menor custo de produção e menores tarifas para o consumidor final. $O$ foco na qualidade na distribuição de eletricidade está relacionado à confiabilidade que a concessionária transmite ao consumidor (Billinton et al., 1997; Araújo et al., 2011; Bandeira, 2012).

De acordo com Slootweg e Enexis (2009), as TICs que integram as Smart Grids transformarão as redes de energia tradicionais em inteligentes, haja vista estas últimas funcionarem de forma unidirecional, ou seja, a transmissão da energia gerada pela central é fornecida em uma única via ao consumidor final, já nas Smart Grids, o fluxo de energia opera de forma bidirecional.

A rede unidirecional de energia é sujeita a falha pois, caso haja defeito durante alguma etapa do fluxo de distribuição energia, ocorrerá um "efeito cascata", onde não será possível contornar a rota da transmissão até consumidor. Além de que, a central só conseguirá solucionar o problema ocorrido, caso seja registrado queixas por parte do consumidor. De outra forma, o fluxo bidirecional de energia permite isolar a área que apresente falha na distribuição de energia, bem como continua a fornecer energia por outros caminhos na rede (Lopes et al., 2012; Costa, 2014).

Lopes et al. (2012) sintetizam os principais componentes de uma Smart Grid, com uma infraestrutura multidirecional, que são: comunicação integrada, fontes de energia alternativas, microgrids, sensoriamento e veículos elétricos. Ribeiro (2011) diz que o conjunto das tecnologias serão os responsáveis pela melhora de vários aspectos técnicos no fornecimento de energia, tais como maior ganho na eficiência energética, melhoria na fiscalização, viabilização técnica do consumo programado e inteligente.

\section{METODOLOGIA}

A pesquisa é um "processo formal e sistêmico de desenvolvimento do método científico. $O$ objetivo fundamental da pesquisa é revelar as respostas para problemas mediante o emprego de procedimentos científicos" (Silva \& Menezes, 2005, p.19). O paradigma desta pesquisa é o funcionalista, com enfoque qualitativo e delineamento descritivo e analítico. Os dados foram levantados por meio de revisão bibliográfica e entrevistas semiestruturadas, triangulados e analisados mediante técnica de análise de conteúdo.

Justifica-se a escolha do paradigma funcionalista pelas características pesquisadas serem empíricas e poderem ser identificadas, estudadas e medidas (Burrell \& Morgan, 1979), e do enfoque qualitativo por ele representar uma maneira de se entender um fenômeno social (Richardson, 1999).

O delineamento descritivo é caracterizado como "a descrição das características de determinada população ou fenômeno ou, então, o estabelecimento de relações entre variáveis" (Gil, 
2002, p.42). Já o analítico visa criar uma teoria aceitável acerca das características que compõem um fenômeno, ao identificar os fatores que contribuem para sua ocorrência ou que o determina (Santos, 2007).

Para levantar os modelos de gestão inteligente de energia elétrica, foi realizada revisão bibliográfica, que segundo Santos \& Candeloro (2006, p. 43) "também é denominada de revisão de literatura ou referencial teórico" e que "revela explicitamente o universo de contribuições científicas de autores sobre um tema específico".

A entrevista, por sua vez, é "uma comunicação verbal entre duas ou mais pessoas, com grau de estruturação previamente definido, cuja finalidade é a obtenção de informações de pesquisa" (Dencker, 2007, p. 165). As entrevistas semiestruturadas são conduzidas por meio de um roteiro previamente estabelecido, contudo, sem demasiada rigidez, o que confere ao entrevistador a liberdade de explorar mais amplamente algumas questões (Richardson, 1999). E a triangulação de dados é uma técnica pela qual é possível investigar um mesmo fenômeno por meio de vários métodos, e assim, determinar a posição do objeto de estudo a partir de diversos pontos de referência (Vergara, 2005).

Os textos que compuseram o referencial teórico foram obtidos por meio das plataformas de pesquisa Periódicos CAPES, ResearchGate, IEE Explore e Google Acadêmico, utilizando-se as seguintes palavras chaves: Urbanização; Smart City; Desenvolvimento sustentável; ODS; modelo de gestão de energia; e Smart Grid. Por fim, os dados encontrados foram analisados por meio da técnica de análise de conteúdo. Segundo Vergara (2005), a técnica de análise de conteúdo atenta para descrições das características encontradas, bem como por identificar e analisar tipos, qualidades e distinções nos textos, além de estabelecer conexões entre elementos estatísticos e análise qualitativa dos materiais.

\section{RESULTADO E DISCUSSÃO}

Com a finalidade de atingir o primeiro objetivo específico, foi levantado, a partir de revisão da literatura, os principais modelos de gestão de energia e ferramentas teóricas que contribuem com a gestão energética, que são o Balanced Scorecard (BSC), o Sistema de Gestão de Energia (Norma ISO 50001:2011), o Plan-Do-Check-Act Cycle (PDCA) e o Modelo de Excelência em Gestão (MEG) (Godini, 2017).

O primeiro modelo de gestão encontrado foi o "Balanced Scorecard (BSC)", que é muito utilizado nas empresas como ferramenta de medição de desempenho (Pinto et al., 2020). Pinto et al. (2020, p. 567) consideram que o BSC contribui com a elaboração de estratégias e a sua disseminação dentro da empresa, ao realizar a avaliação dentro de dimensões temporais: "passado (depois das ações), presente (durante a execução das mesmas) e futuro (antes da sua implementação), além da comunicação dos indicadores para todos os envolvidos da organização". Ao considerar a proposição de um sistema de gestão inteligente para os serviços de produção e distribuição de energia, o BSC colabora com a definição estratégica do modelo, bem como sua comunicação e avaliação na organização. 
O segundo modelo, nominado de Sistema de Gestão de Energia (ISO 50001:2018), foi criado com o objetivo de incentivar as organizações a produzir e a consumir energia de forma inteligente e sustentável, especificando quais são os requisitos necessários que uma empresa deve seguir para estabelecer, implementar, manter e melhorar o desempenho do sistema de gestão de energia, assegurando as devidas orientações de uso consciente sobre o uso de energia (Frozza et. al., 2012; ABNT, 2018).

De acordo com Morabito (2017), a ISO 50001 tem como base a metodologia Plan-Do-CheckAct Cicle (PDCA). O "ciclo PDCA" é modelo de gestão que possui quatro passos, no qual orienta o controle e a melhoria contínua das atividades dentro da empresa (Girardi, 2020).

O último modelo, Modelo de Excelência em Gestão (MEG), "estimula o alinhamento, a integração, o compartilhamento e o direcionamento em toda a organização, para que atue com excelência na cadeia de valor e gere resultados a todas as partes interessada" (FNQ, 2020, p.1). O MEG tem como característica o modelo sistêmico, inspirado no ciclo Plan, Do, Check, Act (PDCA); não possui prescrição em suas práticas de gestão; e é adaptável a todo tipo de organização.

Diante dos 4 modelos expostos, é possível aperceber que as empresas públicas e privadas estão em busca de modelos de gestão para melhorar sua gestão estratégica da rede de energia, haja vista a alta competitividade do setor. São necessárias estratégias empresariais diferenciadas para proporcionar às organizações novos caminhos de crescimento e de sustentabilidade empresarial, ao melhorar a definição e o gerenciamento de suas estratégias e dos recursos disponíveis e utilizados (Moraes, 2017; Zaluski et al., 2019;).

Por meio da literatura, também foram encontrados modelos de infraestrutura energética, que são: modelo energético tradicional (Figura 1) e modelo de infraestrutura de uma Smart Grid (Figura 2).

\section{Figura 1}

\section{Modelo energético unidirecional}

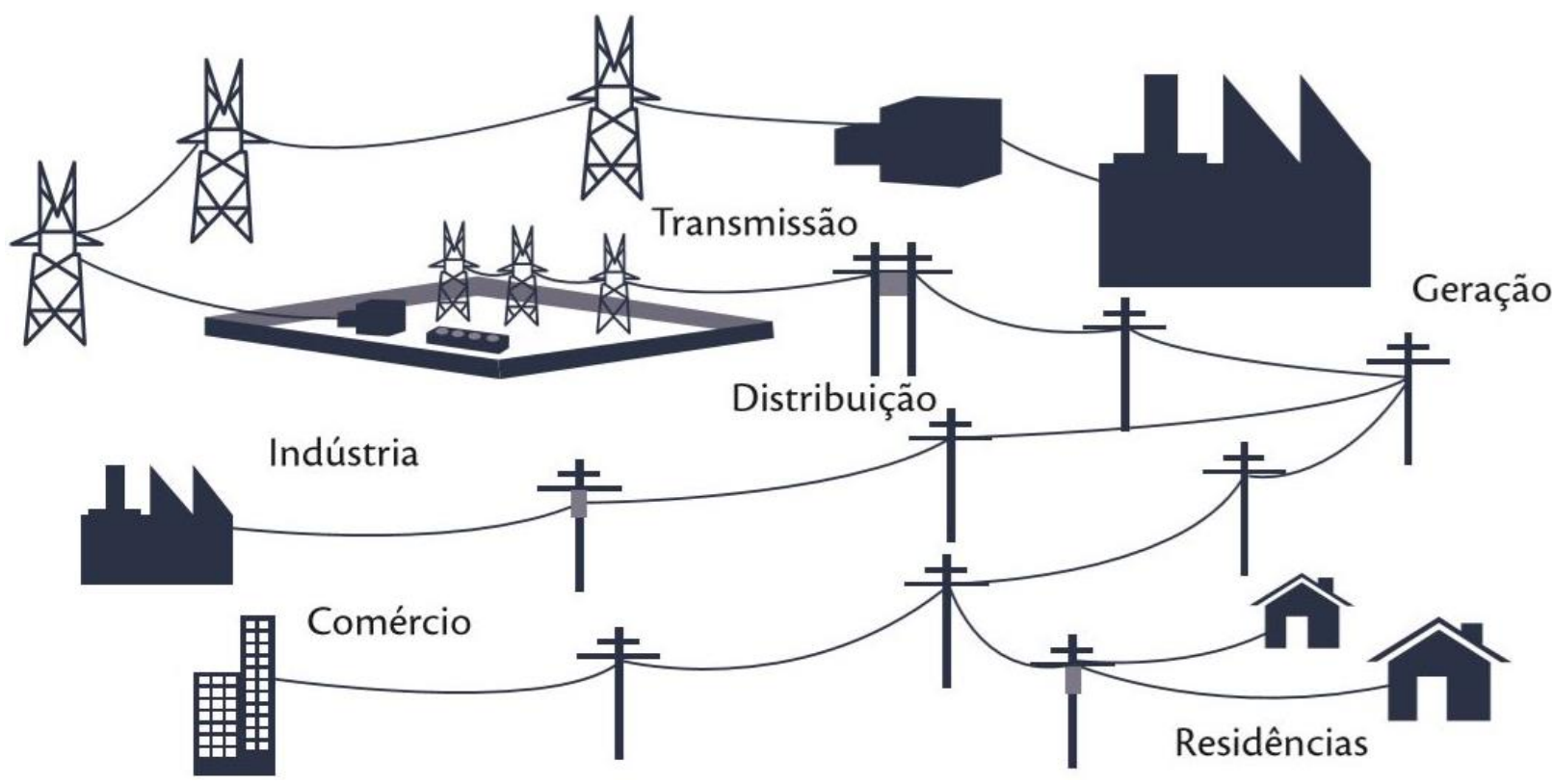

Fonte: Yu et. al. (2011, p.50). 
$\mathrm{Na}$ Figura 1, apercebe-se uma distribuição unidirecional de energia, o que significa que se em algum ponto da rede houve interrupção, os outros pontos também sofrem com blecaute. De outra forma, a Smart Grid (Figura 2) possibilita a interrupção de energia em apenas um ponto da rede que apresente problema e, automaticamente, essa região que foi isolada passa a receber energia de outro nó da rede. A manutenção da rede energética se dará em seguida, haja vista o próprio sistema informar a necessidade de reparo, pois informações de interrupções em tempo real são fornecidas tanto para o consumidor quanto para a prestadora do serviço.

\section{Figura 2}

Modelo de uma Infraestrutura de uma Smart Grid.

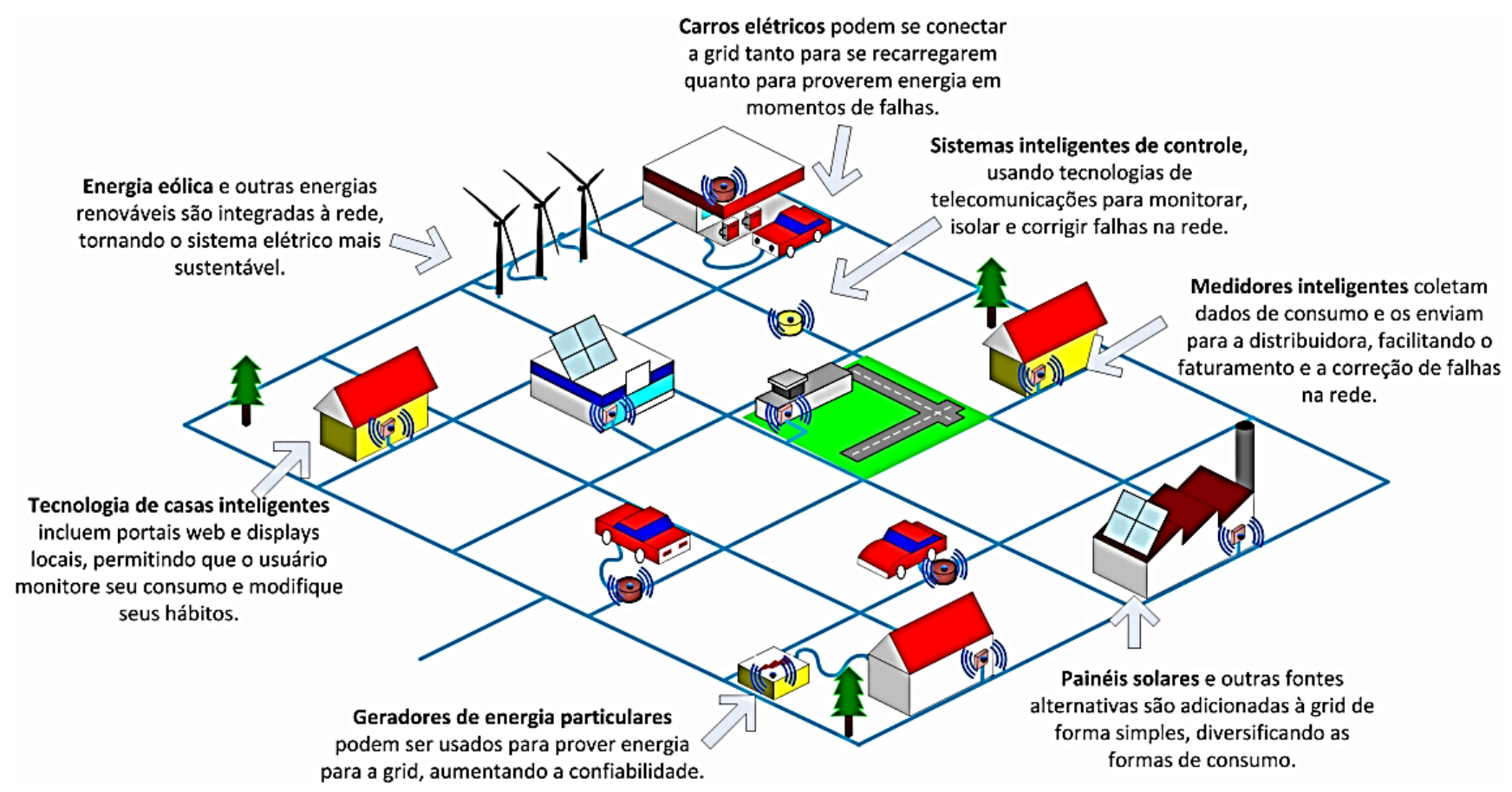

Fonte: Lopes et al. (2012, p.9).

É possível observar a diferença entre os modelos, no primeiro (Figura 1), a energia é gerada somente pela central e transmitida em via única até o consumidor final, diferentemente do segundo modelo (Figura 2), no qual há uma infraestrutura multidirecional, ou seja, o consumidor final não depende de apenas uma fonte de energia, pois tem acesso a diversas fontes de energia renováveis, além de medidores inteligente que interliga o consumidor a rede, proporcionando a este 0 gerenciamento do seu consumo em tempo real (Slootweg \& Enexis, 2009; DECC, 2012; Lopes et al., 2012).

A Smart Grid, dessa forma, contribui com o desenvolvimento sustentável em todos os aspectos teóricos do Triple Bottom Line (ambiental, social e econômico). Quanto ao aspecto ambiental, a Smart Grid, ao inserir em sua rede energética fontes de energia renováveis, como a energia solar e a eólica, e ao combinar seus usos como modos tradicionais de geração de energia, otimiza o uso da rede energética e favorece o consumo de fontes renováveis. 
Para atingir o segundo objetivo específico, foi elaborado um modelo teórico de Smart Grid (Figura 3), a partir da revisão da literatura. A partir da Figura 3 é possível vislumbrar que a Central de Controle é interconectada por sensores inteligentes, voltados a detectar possíveis falhas que podem ocorrer durante o processo de transmissão e distribuição de energia. A geração de energia, por meio fontes de energia tradicionais, já utilizadas pelas concessionárias, juntamente com a energia renovável (eólica, solar ou hidrelétrica), é o diferencial do sistema sustentável.

\section{Figura 3}

Modelo teórico de Smart Grid

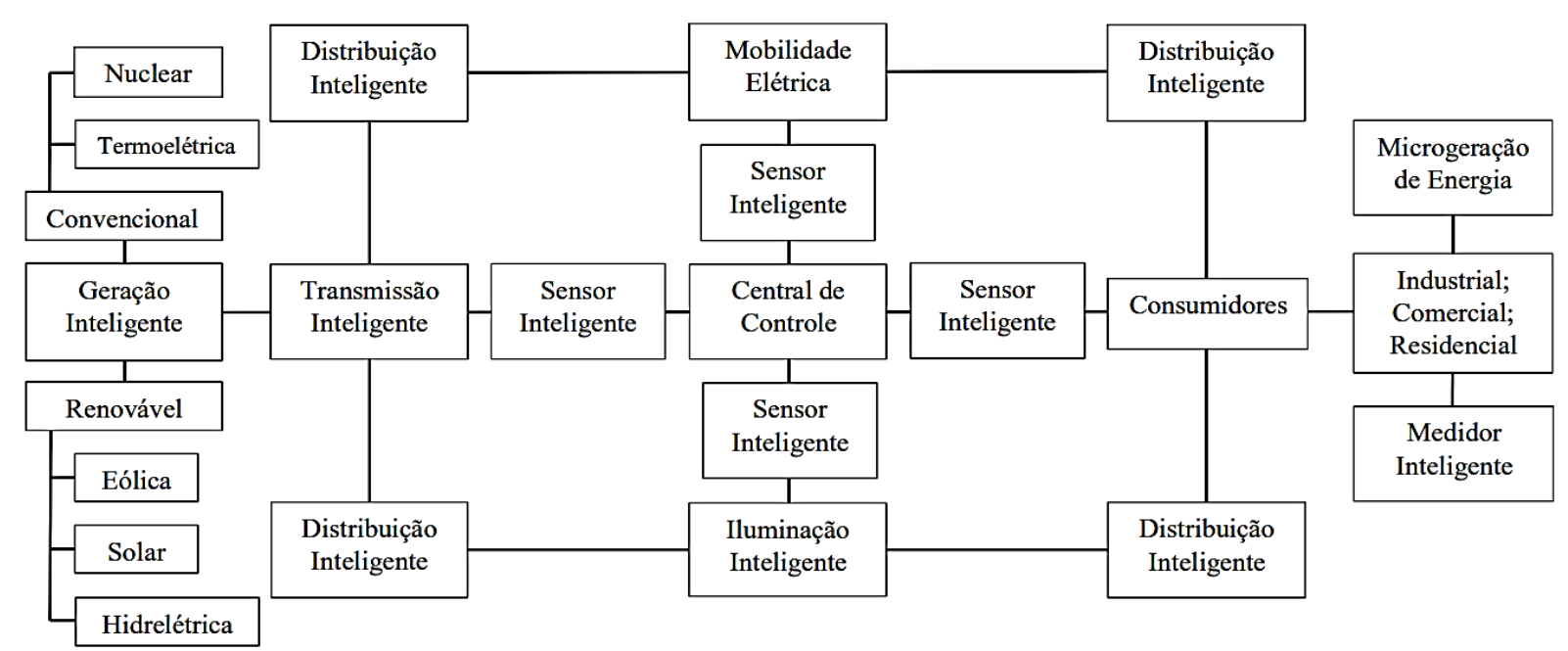

Nota. Elaborado pelos autores.

A Figura 3 se refere ao contexto de transição da geração de energia elétrica por meio de fontes não renováveis e renováveis conjuntamente, em vez de utilizar apenas fontes não renováveis. Solução esta que poderia servir a Parintins/AM, voltado à transição sustentável da produção de energia na municipalidade. O processo de transmissão e distribuição de energia, de forma inteligente, pelo sistema, também foi destacado na Figura 3, voltado aos vários usos residenciais, urbanos e industriais. No sistema inteligente, estes usuários podem controlar o seu consumo por meio dos medidores inteligentes, e ainda, produzir sua própria energia com a microgeração.

O terceiro e quarto objetivos específicos foram atingidos por meio de uma entrevista com o coordenador local da concessionária "Oliveira Energia" de Parintins/AM e com o técnico de energia desta urbe, bem como ao considerar os aspectos já descritos e analisados nos objetivos específicos um e dois. As entrevistas foram conduzidas por meio de um roteiro de entrevista composto por 36 (trinta e seis) questões semiestruturadas. O coordenador local ficou responsável por responder questões que abrangesse a gestão da empresa, e o técnico pela parte operacional.

Durante a entrevista foi perguntado sobre a OD7 da "Agenda 2030", e o coordenador citou que até 2024 irá passar a "Linha de Transmissão 230Kv (Oriximiná-Juruti-Parintins e Subestações Associadas) em Parintins/AM, com o objetivo de eliminar integralmente a produção de energia termoelétrica nesta municipalidade, a qual passará a ser abastecida por uma fonte energia "hidrelétrica", que é renovável. Foi possível aperceber, também, a preocupação do gestor com a 
acessibilidade de energia quando o Coordenador citou o "Programa Luz para Todos", o qual levará energia para as comunidades do interior de Parintins/AM, cujo cadastro pode ser feito pelo site da empresa.

Até 2024 vai passar uma transmissora, o Linhão de Tucuruí. Vai passar aqui justamente para eliminar a queima de diesel. A ideia e de tornar a energia mais sustentável, porque vai ser uma hidrelétrica. [...]". (Coordenador Local Oliveira Energia).

A luz para todos cadastra um trecho que ainda não tem energia e segue um cronograma [...] agora existe um site que o morador da zona rural pode entrar e se cadastrar. (Coordenador Local Oliveira Energia).

O coordenador informou que dentro da repartição não é tratado assuntos sobre Smart City e Smart Grids, pois a empresa ainda está passando por uma fase de reestruturação, devido a privatização da Amazonas Energia. Em relação a gestão da empresa, é utilizado o modelo de gestão PDCA adaptado à realidade local e seguindo os regulamentos da ANEEL, o qual se chama "Sexta Estratégica". Nela, são conduzidas reuniões estratégicas para estabelecimento de metas e verificação os acertos e erros durante a semana.

Aqui na empresa não é tratado, mas no futuro sim, porque no momento estamos passando por uma fase de reestruturação [...] estamos passando por um processo de estabelecimento de metas com os colaboradores. (Coordenador Local Oliveira Energia).

Nossa estratégia é bem parecida com o PDCA, o qual é adaptado a nossa realidade, pois precisamos seguir o que a ANEEL impõe. Como as reuniões são as sextas-feiras, chamamos de 'Sexta estratégica. (Coordenador Local Oliveira Energia).

Percebeu-se, a partir das entrevistas, a limitação ao acesso às informações documentadas, por falta de transparência, na divulgação dos dados da Oliveira Energia. Ainda, foi informado pelo entrevistado técnico que em Parintins/AM a rede elétrica possui principalmente características de uma rede convencional de energia: a) fluxo de comunicação unidirecional de energia; b) geração de energia maiormente centralizada e de fonte não renovável (termoelétrica); c) geração de energia fotovoltaica, renovável, em parcos lugares; d) poucos sensores na rede; e) manutenção realizada manualmente; $f$ ) leitura dos medidores manual.

A partir da descrição supra acerca do modelo hodierno da gestão de energia elétrica em Parintins/AM, bem como das expectativas futuras de sua reconfiguração (objetivo específico 3), é possível elencar dois fatores impulsionadores à implantação de uma Smart Grid nesta urbe, que é o início da geração de energia fotovoltaica na cidade, mesmo que ainda pouco expressiva, e a possibilidade de se utilizar energia hidroelétrica, renovável, para abastecer a cidade e, como fatores limitantes, destaca-se a precária gestão e operação da concessionária de energia nesta municipalidade e baixa divulgação de informações acerca da operação e gestão da Oliveira Energia. Este parágrafo se refere ao alcance do objetivo específico quatro elencado. 


\section{CONCLUSÃO}

Ao considerar a pergunta de pesquisa formulada, "Como as Smart Grids se configuram em alternativa de desenvolvimento inteligente e sustentável em Parintins/AM?", identificou-se que as Smart Grids se configuram em alternativa de desenvolvimento inteligente e sustentável em Parintins/AM nos aspectos da infraestrutura de geração e transmissão de energia.

Quanto a geração de energia, a implantação de uma Smart Grid favoreceria utilização de fontes de energia renováveis na grid energética da municipalidade, oriunda de fontes diversas, com consequente redução do impacto ambiental hodiernamente gerado pela queima de combustíveis fósseis (emissão de $\mathrm{CO}_{2}$ ) para fornecimento de energia nesta urbe.

No que se refere à transmissão de energia, os medidores inteligentes serviriam à população e à concessionária de energia como modo de acompanhar o consumo de energia em tempo real. Os dados de consumo energia monitorados em tempo real pela concessionária, ao serem analisados por meio de sistemas computadorizados, auxiliariam na previsão de demanda e identificação de picos de consumo, o que favorece a elaboração de campanhas de uso consciente de energia, como menores tarifas fora dos horários de pico.

Ao analisar os benefícios para a população, o acompanhamento em tempo real do consumo de energia auxilia no controle dos custos despendidos com esse serviço. Em horários de pico de consumo, por exemplo, quando a tarifa energética tem preço a maior, os consumidores são informados em tempo real do custo da tarifa energética e tem estímulo negativo ao uso da rede. Outrossim, quando os medidores inteligentes são associados às residências inteligentes, novas combinações de usos de energia ainda são ampliadas, como a programação para que eletroeletrônicos e eletrodomésticos funcionem fora dos momentos de pico no consumo de energia.

Os sensores dos fluxos de energia na grid também reforçam a percepção das Smart Grids como alternativa de desenvolvimento inteligente e sustentável, haja vista que o fluxo bidirecional de energia evita apagões, o que favorece o fornecimento ininterrupto de energia na municipalidade.

Apercebe-se, assim, que as Smart Grids se configuram em alternativa de desenvolvimento sustentável em Parintins/AM nos aspectos econômicos (acompanhamento dos custos de energia em tempo real pela concessionária de energia e consumidores), ambientais (redução da pegada de carbono da concessionária de energia) e sociais (distribuição de energia initerruptamente na municipalidade).

Ainda, este estudo identificou que a gestão inteligente de energia não é uma realidade do município de Parintins/AM. A precariedade de informações resultante das entrevistas quanto a aspectos básicos da gestão do sistema de energia desta municipalidade indica falta de conhecimento técnico da administração e da operação do serviço de fornecimento de energia pela concessionária Oliveira Energia, o que impacta negativamente na formulação de um planejamento estratégico de médio e longo prazo voltado à transição da geração e distribuição de energia para um modelo inteligente.

As limitações do estudo são oriundas da precariedade das respostas dos entrevistados, todavia, essa limitação constitui-se em um dos achados do estudo, que identificou a debilidade da 
gestão executiva e operacional da concessionária de energia desta urbe, que é um serviço vital para a prosperidade econômica, social e ambiental de qualquer região. Como sugestões de pesquisas futuras, sugere-se o levantamento de como se dá a gestão executiva e operacional do fornecimento de energia elétrica nas outras cidades da região do baixo Amazonas, a fim de identificar se essa problemática é exclusiva de Parintins ou se é um traço da forma de gestão de serviços de energia na região.

\section{REFERÊNCIAS}

Associação Brasileira de Normas Técnicas [ABNT]. (2018). ABNT NBR ISO 50001: Sistemas de gestão de energia - Requisitos com orientação para uso. Rio de Janeiro: ABNT.

Ahvenniemi, H., Huovila, A., Pinto-Seppä, I. \& Airaksinen, M. (2017). What are the differences between sustainable and smart cities?. Cities, 60, 234-245.

Alto, P. (2008). The green grid: energy savings and carbon emissions reductions enabled by a smart grid. California: EPRI.

Agência Nacional de Energia Elétrica [ANEEL]. (2019). Contrato de concessão da Amazonas Energia. Disponível em: http://www.aneel.gov.br/sala-de-imprensa/lasset publisher/zXQREz8EVIZ6/content/id/18408843.

Angelidou, M. (2014). Smart city policies: A spatial approach. Cities, 41, S3-S11.

Araújo, A. N. B. D., Fernandes, H. T. P., Gomes, J. P. B. D. S., Morais, A. V. D., Queiroz, J. V., \& Vasconcelos, N. V. C. D. (2011). Gestão da Informação e o Sistema Smart Grid: Um Estudo de Caso da UFRN. II Congresso Internacional IGLU, Florianópolis.

Bandeira, F. P. M. (2012). Redes de energia elétrica inteligentes (Smart Grids). Nota técnica. Consultoria Legislativa. Disponível em: https://web.bndes.gov.br/bib/jspui/bitstream/1408/2927/1/RB\%2040\%20Redes\%20el\%C3\%A 9tricas\%20inteligentes P.pdf.

Barbosa, G. B., Urquiza, M. F., Cândido, M. B. B. \& Pugliesi, J. B. (2013). Tecnologia integrada às áreas para o desenvolvimento de cidades inteligentes. Revista Eletrônica de Sistemas de Informação e Gestão Tecnológica, 3(1).

Billinton, R., Salvaderi L., McCalley J. D., Chao, H., Seitz, T., Allan, R.N., Odom, J. \& Fallon C. (1997). Reliability issues in today's electric power utility environment. IEEE Transactions on Power System, 12(4). Doi: 10.1109/59.627880.

Burrell, G. \& Morgan, G. (1979). Sociological paradigms and organisational analysis. London: Heinemann Educational Books. ISBN: 9781857421149.

Cavalcante, N. W. F. \& Tavares, E. (2017). Métodos de Avaliação de Projetos de Smart Cities: Um Estudo Teórico. XX SEMEAD - Seminários em Administração.

Chourabi, H., Nam T. \& Walker S. (2012). Understanding smart cities: An integrative framework. In 2012 45th Hawaii international conference on system sciences. IEEE. Doi: 10.1109/HICSS.2012.615.

Costa, S. A. H. (2014). Os desafios da implantação da smart grid no modelo de negócios do setor elétrico: proposta de configuração de uma rede inteligente. Dissertação de Mestrado. 
Department of Energy \& Climate Change [DECC]. (2012). Smart meters: a guide. Disponível em: http://www.decc.gov.uk/en/content/cms/tackling/smart meters/smart meters.aspx.

Dencker, A. F. M. (2007). Pesquisa em turismo: planejamento, métodos e técnicas. 9. ed. São Paulo: Futura.

Falcão, D. (2009). Smart grids e microrredes: o futuro já é presente. VIII Simpase, Simpósio de Automação de Sistemas Elétricos, Programa de Engenharia Elétrica. COPPE: UFRJ.

Fan, Z., Kulkarni, P., Gormus, S., Kalogridis, G., Sooriyabandara, M., Zhu, Z., Lambotharan S. \& Chin W. H. (2013). Smart grid communications: Overview of research challenges, solutions, and standardization activities. IEEE Communications Surveys \& Tutorials, 15(1). Doi: 10.1109/SURV.2011.122211.00021.

Ferreira, M. L., Oliveira, A. de, Cortese, T. T. P. \& Kniess, C. T. (2015). Cidades inteligentes e sustentáveis: problemas e desafios. In. Benini, N. S. M. \& Rosin, J. A. R. G. (Orgs). Estudos Urbanos: uma abordagem interdisciplinar da cidade contemporânea. Tupã: São Paulo.

Fundação Nacional da Qualidade [FNQ]. (2020). O que é MEG? São Paulo: Fundação Nacional da Qualidade.

FRAGMAQ. (2015). Conheça as vantagens e desvantagens da usina termoelétrica. Disponível em: https://www.fragmaq.com.br/blog/conheca-vantagens-desvantagens-usina-termoeletrica/.

Frozza, J. F., Lafay J. S., Baldin, V. \& Marangoni, F. (2012). Metodologia de Implantação de um sistema de Gestão de Energia Utilizando ABNT NBR ISO 50001. In Congresso Nacional de Excelência em Gestão, VIII. Rio de Janeiro.

Gil, A. C. (2002). Como elaborar projetos de pesquisa. São Paulo: Atlas.

Gil-Garcia, J. R., Pardo, T. A. \& Nam, T. (2015). What makes a city smart? Identifying core components and proposing an integrative and comprehensive conceptualization. Information Polity, 20(1), 61-87.

Girardi, G. (2020). Gestão de energia: o que é e como fazer? Disponível em: https://www.way2.com.br/blog/gestao-de-energia.

Glaeser, E. L. \& Berry, C. R. (2006). Why are smart places getting smarter? Taubman Center Policy Brief. Harvard University: Taubman Center.

Godini, C. (2017). Modelos de gestão como ferramentas para a otimização. Disponível em: http://www.engenhariaearquitetura.com.br/2017/12/modelos-de-gestao-como-ferramentaspara-otimizacao.

Guedes, A., Mota, J., Tavares, M. \& Abreu R. (2015). Cidades Inteligentes-Smart CitiesInfraestrutura tecnológica: caracterização, desafios e tendências. Projeto FEUP, 2015.

Hollands, R. G. (2008). Will the real smart city please stand up? Intelligent, progressive or entrepreneurial?. City, 12(3), 303-320.

Instituto Brasileiro de Geografia e Estatística [IBGE]. (2020). População estimada, 2020. Disponível em: https://cidades.ibge.gov.br/brasil/am/parintins/panorama.

International Energy Agency [IEA]. (2011). Smart Grid - Smart Customer Policy Needs. Workshop Report: Energy Efficiency Working Party. 
Instituo de Pesquisa Econômica Aplicada [IPEA] (2019a). Cadernos ODS 7. Disponíve em: https://brasil.un.org/pt-br/sdgs/7.

Instituo de Pesquisa Econômica Aplicada [IPEA] (2019b). Cadernos ODS 11. Disponível em: https://brasil.un.org/pt-br/sdgs/11.

Innovation-Observatory. (2012). Smart Grid Technology Investment: Forecasts for 2012-2030. ISBN13: 978-0957456600.

Lima. A. J. S., Pinheiro, A. M., Natividade, J. S. \& Rocha, M. Q. (2013). Festival Folclórico de Parintins: 100 anos de histórias para contar.

Lopes, Y., Franco, R. H. F., Molano, D. A., Santos, M. A., Calhau, F. G., Bastos, C. A. M., Martins, J. S. B. \& Fernandes, N. C. (2012). Smart Grid e IEC 61859: Novos Desafios em Redes e Telecomunicações para o Sistema Elétrico. XXX Simpósio Brasileiro de Telecomunicações.

Morabito, G. F. (2017). Proposição de indicadores e métricas para avaliar e mensurar a eficiência das subestações de um microgrid. Dissertação. PUC: Rio de Janeiro.

Moraes, R. R. (2017). Planejamento estratégico no setor público: metodologia de implementação a partir do BSC. Projeto de investigação científica do Curso de Administração. Centro Universitário Fibra: Belém.

Northeast-Group, L. (2012). Brazil Smart Grid: Market Forecast (2012 - 2022). Smart metering (AMI), distribution automation, wide area measurement, home energy management. Northeast Group Ilc.

Pinto, S. H. B., Tonini, A. C., Yoo, N. S. L., \& Katayose, E. M. (2020). Gestão estratégica através do Balanced Scorecard (BSC): implantação em empresas brasileiras. Brazilian Journal of Business, 2(1), 564-580.

Ribeiro, C. L. (2011). Aspectos institucionais para o smart grid no Brasil: riscos, oportunidades e desafios regulatórios. Brasília: Universidade de Brasília.

Richardson, R. J., Peres, J. A. S., Wanderley J. C. V., Correia L. M. \& Peres. M. H. M. (1999). Pesquisa Social: métodos e técnicas. 3. ed. São Paulo: Atlas.

Rivera, R., Esposito, A. S. \& Teixeira, I. (2013). Redes elétricas inteligentes (smart grid): oportunidade para adensamento produtivo e tecnológico local. Revista do Banco Nacional de Desenvolvimento Social - BNDES, Rio de Janeiro n. 40, p. 43-83.

Santos, V. \& Candeloro, R. J. (2006). Trabalhos acadêmicos: uma orientação para a pesquisa e normas técnicas. Porto Alegre: AGE.

Santos. A. R. (2007). Metodologia científica: a construção do conhecimento. Rio de Janeiro: Lamparina.

Silva, E. L. \& Menezes, E. M. (2005). Metodologia da pesquisa e elaboração de dissertação. 4 ed. Florianopolis: UFSC.

Slootweg, H. \& Enexis, B. V. (2009). Smart Grids-the future or fantasy?. In 2009 IET Smart MeteringMaking It Happen. IET. p. 1-19, doi: 10.1049/ic.2009.0060.

Organização das Nações Unidas [ONU]. (1945). Carta das Nações Unidas. Disponível em: https://brasil.un.org/sites/default/files/2021-08/A-Carta-das-Nacoes-Unidas.pdf.

Vergara, S. C. (2005). Métodos de coleta de dados no campo. São Paulo: Atlas. 
Word Commission on Environment and Development [WCED]. (1987). Our common future. Oxford: Oxford University Press.

Yu, X., Cecati, C., Dillon, T., \& Simoes, M. G. (2011). The new frontier of smart grids. IEEE Industrial Electronics Magazine, 5(3), 49-63. Doi: 10.1109/MIE.2011.942176.

Zaluski, F. C., Hedlund, P.R., Sausen, J. O. \& Thesing, N. J. (2019). A utilização de indicadores gerenciais como fator de aumento da capacidade de escolha estratégica: um estudo de caso no setor elétrico. Revista Contribuciones a las Ciencias Sociales (abril, 2019). Disponível em: https://www.eumed.net/rev/cccss/2019/04/aumento-capacidade-escolha.html. 